\title{
LA ÚLCERA PÉPTICA: ¿UN TRASTORNO GASTROINTESTINAL INFECCIOSO O BIOPSICOSOCIAL?
}

\author{
SERGIO TOBÓN ${ }^{1}$, BONIFACIO SANDÍN ${ }^{2}$ y STEFANO VINACCIA ${ }^{3}$ \\ ${ }^{1}$ Universidad de Antioquía, Colombia \\ ${ }^{2}$ Universidad Nacional de Educación a Distancia, España \\ ${ }^{3}$ Universidad de San Buenaventura, Colombia
}

\begin{abstract}
La úlcera péptica (UP) es entendida en la actualidad básicamente como un trastorno gastrointestinal de tipo infeccioso, causado por la bacteria $H$. pylori, la cual se descubrí en el estómago de los seres humanos en el año de 1982. Esto ha llevado a que la investigación se centre en los factores biológicos, dejando de ser tenidos en cuenta los factores psicosociales, los cuales tradicionalmente se han asociado con esta condición médica. El objetivo del presente artículo es presentar un análisis del papel de la $H$. pylori y del estrés psicosocial en la UP, planteando la necesidad de establecer un modelo biopsicosocial, con el fin de facilitar el conocimiento de su etiología y mejorar su tratamiento.
\end{abstract}

Palabras clave: úlcera péptica, Helicobacter pylori, estrés psicosocial, modelo biopsicosocial, trastornos gastrointestinales.

\section{Peptic ulcer: ¿An infectious or bio psychosocial gastrointestinal disease?}

\begin{abstract}
Peptic ulcer (PU) is currently understood as a gastrointestinal disease of an infectious type, caused by the bacteria Helicobacter pylori, which was discovered in the stomach of the human being in 1982. This has emphasized the research on the biological factors, not taking into consideration psychosocial factors, which have been traditionally associated with this medical condition. The aim of this article is to present an analysis of the role that $H$. pylori and psychosocial stress in the PU, presenting the need to establish a biopsychosocial model, in order to facilitate the knowledge of its etiology and to improve its treatment.
\end{abstract}

Key words: peptic ulcer, Helicobacter pylori, psychosocial stress, biopsychosocial model, gastrointestinal disorders.

\section{INTRODUCCIÓN}

Existe evidencia de que los trastornos gastrointestinales se relacionan de forma muy estrecha con los trastornos psicológicos (Tobón, Vinaccia y Sandín, 2004). La UP es una enfermedad frecuente, ya que cerca del $10 \%$ de la población la puede tener en algún momento de su vida (Soll, 1993). Este trastorno gastrointestinal consiste en una lesión que penetra en la mucosa de la pared gastrointestinal, afectando la musculatura

Correspondencia: Sergio Tobón, Colegio Mayor Nuestra Señora de África, Ramiro de Maeztu s/n, 28040 Madrid. Correo-e: stobon5@yahoo.es subyacente. La lesión ulcerosa puede darse tanto en el estómago (úlcera gástrica, UG) como en el duodeno (úlcera duodenal, UD). Los principales síntomas son el dolor y el ardor en la parte superior de la región abdominal. Igualmente pueden presentarse otros síntomas tales como náuseas, vómitos, eructos e hinchazón abdominal. Debido a que estos síntomas son comunes en otros trastornos gastrointestinales, es necesario realizar una endoscopia digestiva superior con el fin de diagnosticar este problema médico. La patofisiología esencial de la UP se debe a un desequilibrio entre los factores protectores (p.ej., producción de moco y 
bicarbonato) y los factores agresivos que actúan sobre la mucosa gastrointestinal (p.ej., la actividad ácido-péptica).

Marschall y Warren publicaron un estudio en 1983 en el cual informaron del descubrimiento de una bacteria en el estómago. Esta bacteria se denominó luego Helicobacter pylori ( $H$. pylori). Múltiples estudios han demostrado que esta bacteria es un factor de riesgo fundamental en la UP. En este sentido, se ha hallado que cerca del $95 \%$ y $85 \%$ de las personas con UD y UG respectivamente están infectadas, y que la erradicación de la bacteria aumenta la tasa de curación de este problema de salud y reduce de forma significativa las recaídas (Quan y Talley, 2002). Así mismo, se ha podido determinar que hay una relación temporal entre la gastritis asociada a la $H$. pylori y el origen de la UP; que la probabilidad de desarrollar la UP aumenta a mayor exposición a la $H$. pylori, y que hay una serie de mecanismos fisiopatológicos asociados a la bacteria que explican su relación con esta condición médica (p.ej., la inflamación de la mucosa gástrica y las alteraciones en la actividad ácido-péptica) (Gisbert, Boixeda y Martín de Argila, 1996). A raíz de esto, se ha llegado a la concepción de que la UP es fundamentalmente una enfermedad infecciosa.

Antes del descubrimiento del rol patógeno de la $H$. pylori, la UP era considerada como una enfermedad multifactorial, cuya etiología era debida a diversos factores tales como los psicológicos (estrés y características de personalidad), los sociales (condiciones económicas y apoyo social), los genéticos y los comportamentales (hábito de fumar, consumo de alcohol, comer de forma irregular, etc.). Sin embargo, la UP se ha helicobacterizado (Melmed y Gelpin, 1996) y tales factores son escasamente tenidos en cuenta hoy en día tanto en la investigación sobre el tema como en la práctica clínica de los profesionales de la medicina y de la gas- troenterología. El objetivo del presente trabajo es presentar una discusión en torno al papel de la $H$. pylori en la UP, analizando la posible implicación del estrés psicosocial en su etiología y curso, considerando la necesidad de un enfoque biopsicosocial para este trastorno gastrointestinal.

\section{¿ES LA ÚLCERA PÉPTICA UNA ENFERMEDAD INFECCIOSA?}

No hay duda de que la $H$. pylori está implicada en la etiología y curso de la UP, lo que sí es objeto de discusión es que se le considere como el factor etiológico fundamental, en un problema de salud que es altamente complejo. De acuerdo con la revisión de la literatura científica, hay todavía algunos aspectos que no son suficientemente explicados por la teoría infecciosa de la UP. A continuación se describen algunos de ellos.

1. Más de la mitad de la población mundial está infectada por la $H$. pylori y tiene una gastritis histológica asociada (Gisbert et al., 1996), pero sólo un pequeño número de personas infectadas desarrolla la UP, que es cercano al $10 \%$ a lo largo de la vida. Se ha hallado que hay factores de virulencia que podrían explicar este hecho, es decir, que hay cepas de $H$. pylori más patógenas que otras, pero esto no explica por qué cerca de un $80 \%$ o $90 \%$ de las personas infectadas no desarrollan esta condición médica.

2. Hay un porcentaje importante de personas con UP que no tienen $H$. pylori. Por consiguiente, esta bacteria no está implicada en todos los casos de UP. Además, hay países con una altísima prevalencia de la infección y, sin embargo, una reducida tasa de enfermedad ulcerosa (Holcombe, 1992). Esto mismo se ha encontrado 
en Tailandia. Un estudio reciente realizado en este país halló que el estrés y la historia familiar de UP, pero no la $H$. pylori, fueron importantes factores de riesgo para la UP (Wachirawat et al., 2003).

3. Hay algunos aspectos relacionados con la incidencia y la prevalencia de la UP que no están claramente explicados por la bacteria, tales como: (a) la infección por la $H$. pylori es similar en ambos sexos, pero de acuerdo a los estudios epidemiológicos la UD tiene una incidencia significativamente más alta en los hombres; (b) se ha hallado que la prevalencia de la infección aumenta con la edad, pero la enfermedad ulcerosa se caracteriza por una historia autolimitada en el tiempo; (c) la bacteria no explica por qué es mayor la incidencia y prevalencia de las úlceras duodenales en comparación con las gástricas, y (d) no está claro por qué la UP tiene una mayor incidencia en determinadas estaciones del año.

De esta forma, es desde todo punto de vista evidente que la bacteria tiene un rol etiológico fundamental en la UP, siendo por lo general necesaria para que ésta se produzca (en la mayoría de las personas), pero la bacteria por sí misma no explica esta enfermedad. Por tanto, no se puede afirmar que la UP sea exclusivamente una enfermedad infecciosa. Es necesario considerar la implicación de otros factores y establecer su posible interacción con la bacteria en la etiología y curso de esta condición médica.

\section{¿LA ÚLCERA PÉPTICA ES CAUSADA POR FACTORES PSICOPATOLÓGICOS?}

En el campo de la psicología clínica, la psiquiatría y la medicina psicosomática tradicionalmente ha existido la tendencia a considerar la UP como un trastorno gastrointestinal estrechamente asociado a determinados factores psicopatológicos y al estrés psicosocial. Es por ello que se le ha considerado como un problema de salud vinculado con el estilo de vida de las personas y relacionado con el funcionamiento emocional. La concepción psicosomática de la UP parte de algunos estudios clínicos basados en la aplicación del método psicoanalítico. Alexander (1950) llegó a la formulación de que la UP era una condición médica causada por conflictos emocionales inconscientes específicos reprimidos, los cuales, a través del sistema nervioso autónomo y a partir de dificultades del contexto, ocasionaban este problema de salud. El conflicto intrapsíquico, según este autor, consiste en una frustración de amor y de cariño durante la infancia, llevando a la necesidad de dependencia en la vida adulta. Es por ello, que a las personas con UP se les ha considerado como de personalidad dependiente. La teoría de Alexander ha tenido una gran influencia en la investigación sobre los factores asociados a los trastornos gastrointestinales y hasta hace dos décadas fue el enfoque predominante en esta área. Aunque algunos estudios posteriores han aportado alguna evidencia sobre la asociación de las características de dependencia con la hipersecreción de ácido y el desarrollo de este trastorno gastrointestinal (véase, p.ej., Weiner, Thaler, Reiser y Mirsky, 1957), tales estudios no son concluyentes y tienen deficiencias metodológicas que impide generalizar sus conclusiones, por lo que en el momento actual no se puede plantear que exista un tipo específico de personalidad ulcerosa (Tobón, Sandín y Vinaccia, 2005).

A pesar de que hay una buena cantidad de estudios que han hallado asociación de diversos factores psicológicos con la UP, tales como la ansiedad, la depresión, la alexitimia, el neuroticismo, 
la ira y el apoyo social (Tobón et al., 2005), gran parte de estos han sido retrospectivos y no aportan evidencias concluyentes frente a un rol etiológico de los factores psicológicos en esta condición médica. Por tal motivo, aunque tales estudios son sugestivos, no se pueden tomar como base para decir que la UP es causada por este tipo de variables psicopatológicas. Además, se ha aportado evidencia de que la misma UP afecta el bienestar psicológico y puede influir en el desencadenamiento de alteraciones emocionales (Jess, 1994; Dimenas et al., 1995).

Sin embargo, sí hay una sólida línea de investigación que ha asociado los sucesos vitales con la etiología de la UP. De esta manera, se ha podido constatar de forma clara que las situaciones de guerra y los desastres naturales se asocian a un mayor riesgo de desarrollar este trastorno gastrointestinal. Esto fue lo que ocurrió durante el ataque aéreo a Londres en 1941 , tras el cual aumentaron los casos de UP de forma significativa, o después del terremoto "Hanshin-Awaji» en Japón (Takakura et al., 1997). Así mismo, se ha documentado que la UP es más frecuente en veteranos de guerra que fueron hechos prisioneros en comparación con los veteranos que no lo fueron (Tobón et al., 2005).

Lo anterior se ha confirmado en varios estudios prospectivos realizados en los últimos doce años, los cuales aportan evidencia concluyente de que los sucesos vitales se asocian a un mayor riesgo de desarrollar UP (Medalie, Stange, Zyzanski y Goldbourt, 1992, Levenstein, Prantera, Scribano y Varvo, 1995). Anda et al. (1992), por ejemplo, investigaron una muestra representativa de 4.511 adultos de los Estados Unidos libres de úlcera y les hicieron un seguimiento longitudinal respecto al grado de estrés percibido obtenido en una línea base. Hallaron que el grado de estrés percibido fue altamen- te predictivo del desarrollo de UP en un seguimiento de 13 años. Así mismo, hay evidencia de que los sucesos vitales se asocian a un peor curso de la UP (Levenstein et al., 1996).

Se concluye entonces que hay evidencia de que el estrés psicosocial se asocia con la UP y está implicado en su desarrollo y curso. Sin embargo, no es el único factor implicado en la UP, pues sólo un mínimo porcentaje de las personas estresadas llegan a desarrollar esta condición médica. Esto muestra que el estrés psicosocial requiere interactuar con otros factores para poder tener algún grado de riesgo en este trastorno.

\section{DE LA SIMPLICIDAD A LA COMPLE- JIDAD: HACIA UN MODELO BIO- PSICOSOCIAL DE LA ÚLCERA PÉPTICA}

Analizando la historia del desarrollo de las teorías etiológicas en la UP se encuentra que siempre ha habido una tendencia marcada a abordar este trastorno desde la simplicidad. Durante varias décadas se consideró como una enfermedad netamente psicosomática causada por factores psicológicos de orden psicodinámico inconsciente (Alexander, 1950). Luego comenzó a estudiarse la agregación familiar y la transmisión genética, hasta el punto de que muchos científicos comenzaron a abandonar el paradigma psicosomático e incorporaron el genético, el cual estaba más cercano a la práctica biomédica. Otros investigadores criticaron esta concepción y plantearon que las causas de la UP eran los factores ambientales y comportamentales tales como el tabaco, el alcohol, los AINEs (fármacos antiinflamatorios no esteroideos), las comidas grasosas e irritantes, etc. En los años 70 comienza a tomar auge en el estudio de la etiología de la UP el enfoque del estrés debido a los estudios de Selye, quien lo conceptuó desde la psicofisiología, y a 
los prometedores avances en esta área del grupo de Holmes, quien diseñó una metodología objetiva para evaluar los sucesos vitales estresantes, llegando a asumirse la UP como una enfermedad ligada al estrés. Luego, a finales de los años 80 y a comienzos de los años 90 , toma auge la teoría infecciosa de la UP y esta es la predominante en la actualidad, debido a lo cual para muchos gastroenterólogos es suficiente con erradicar la $H$. pylori para curar este trastorno gastrointestinal.

La $H$. pylori es fundamental para explicar la etiología y curso de la UP, siendo necesaria, por lo general, para que esta condición médica se produzca. Sin embargo, no es como tal suficiente y se requiere considerar la implicación también de los factores genéticos y del estrés psicosocial. Además de esto, es necesario tener en cuenta las condiciones sociales y económicas, las cuales se asocian a una mayor probabilidad de ocurrencia de ciertos sucesos vitales y a una mayor posibilidad de infección por la $H$. pylori debido a peores condiciones de higiene. De esta forma, consideramos que lo más pertinente es abordar la UP como un trastorno multifactorial.

Hipotéticamente, podemos plantear que el estrés psicosocial está asociado con la UP a través de cuatro posibles mecanismos (Tobón et al., 2005): (1) disminuyendo la inmunocompetencia, lo cual podría llevar a mayores efectos patógenos de la bacteria, tal como se ha hallado con otras infecciones; (2) influyendo en la actividad ácido-péptica (aumentándola o disminuyéndola), lo cual es un mecanismo fisiopatológico esencial en la UP, tanto en la UD como en la UG; (3) disminuyendo los mecanismos defensivos de la mucosa, como por ejemplo la producción de moco, la secreción de bicarbonato y la irrigación sanguínea, y (4) originando o aumentando la frecuencia de conductas de riesgo tales como el fumar, el consumir alcohol, el ingerir AINEs y el comer de forma irregular.

\section{CONCLUSIONES}

La $H$. pylori se mueve en el orden de relaciones extremadamente complejas, raras y paradójicas con el organismo humano en permanente flujo (Parsonnet, 1996). Por tanto, hay cabida en esta área para llevar a cabo múltiples aproximaciones y desde diferentes áreas del conocimiento que puedan contribuir también a un mejor conocimiento de su patogenicidad en la UP. Al respecto, el presente trabajo muestra que es posible pensar que el estrés psicosocial puede tener algún papel en el origen, curso y/o presentación clínica de la UP, junto con los efectos demostrados de la $H$. pylori, señalando esto la importancia de comenzar una nueva área de investigación en este campo que vaya más allá de los mecanismos microbiológicos proximales y considere lo distal, al sujeto y su relación consigo mismo, con los otros y su medio, en el marco de su proceso de vida. En esta línea, entonces, es necesario comenzar a pensar e investigar la UP como un problema de salud de tipo biopsicosocial (Vinaccia, Tobón, Sandín y Martínez-Sánchez, 2001) para esclarecer la relación entre los diferentes factores implicados.

\section{REFERENCIAS BIBLIOGRÁFICAS}

Alexander, F. (1950). Psychosomatic Medicine: Its Principles and Applications. New York: W.W. Norton.

Anda, R.F., Williamson, D.F., Escobedo, L.G., Remington, P.L., Mast, E.E., y Madans, J.H. (1992). Self- perceived stress and the risk of peptic ulcer disease. A longitudinal study of US adults. Archives of Internal Medicine, 152, 829-833. 
Dimenas, E., Glice, H., y Hallerback, B. (1995). Well-being and gastrointestinal symptoms among patients Referred to endoscopy owing to suspected duodenal ulcer. Scandinavian Journal of Gastroenterology, 30, 1046-1052.

Gisbert, J.P., Boixeda, D., y Martín de Argila, C. (1996). Infección por Helicobacter pylori y úlcera péptica. En D. Boixeda, J.P. Gisbert y C. Martín de Argila (Eds.), Infección por h. pylori ¿Dónde esta el limite? Barcelona: Prous Science.

Holcombe, C. (1992). Helicobacter pylori: The African enigma. Gut, 33, 429-431.

Jess, P. (1994). Gastric acid secretion in relation to personality, affect and coping ability in duodenal ulcer patients. A multivariate analysis. Danish Medical Bulletin, 41, 100-103.

Levenstein, S., Prantera, C., Scribano, M.L., Varvo, V., Berto, E., y Spinella, S. (1996). Psychological predictors of duodenal ulcer healing. Journal of Clinical Gastroenterology, 22, 84-89.

Levenstein, S., Prantera, C., Scribano, M.L., y Varvo, V. (1995). Patterns of biologic and psychological risk factors in duodenal ulcer patients. Journal of Clinical Gastroenterology, 21, 100-107.

Marshall, B., y Warren, J.R. (1983). Unidentified curved bacilli on gastric epithelium in active chronic gastritis. Lancet, 1, 12731275.

Medalie, J.H., Stange, K.C., Zyzanski, S.J., y Goldbourt, U. (1992). The importance of biopsychosocial factors in the development of duodenal ulcer in a cohort of middle-aged men. American Journal of Epidemiology, 136, 1280-1287.

Melmed, R. N., y Gelpin, Y. (1996). Duodenal ulcer: The helicobacterization of a psychosomatic disease? Israel Journal of Medical Sciences, 32, 211-216.

Parsonnet, J. (1996). Helicobacter pylori in the stomach-A paradox unmasked. The
New England Journal of Medicine, 335, 278-280.

Quan, C., y Talley, N.J. (2002). Management of peptic ulcer disease not related to Helicobacter pylori or NSAIDs. The American Journal of Gastroenterology, 97, 2950-2961.

Soll, H.A. (1993). Gastric, duodenal y stress ulcer. En M. Sleisenger y J. Fordtran (Eds.), Gastrointestinal disease (pp. 580-679). Philadelphia, Pa: WB Saunders.

Takakura, R., Himeno, S., Kanayama, Y., Sonoda, T., Kiriyama, K., Furubayashi, T., Yabu, M., Yoshida, S., Nagasawa, Y., Inoue, S., y Iwao, N. (1997). Follow-up after the Hanshin-Awaji earthquake: diverse influences on pneumonia, bronchial asthma, peptic ulcer and diabetes mellitus. Internal Medicine, 36, 87-91.

Tobón, S., Sandín, B., y Vinaccia, S. (2005). Trastornos gastrointestinales: psicopatología y tratamientos psicológicos. Madrid: Klinik.

Tobón, S., Vinaccia, S., y Sandín, B. (2004). Modelo procesual del estrés en la dispepsia funcional: implicaciones para la evaluación y el tratamiento. Revista de Psicopatología y Psicología Clínica, 9, 81-98.

Vinaccia, S., Tobón, S., Sandín, B., y Martínez-Sánchez, F. (2001). Estrés psicosocial y úlcera péptica duodenal: una perspectiva biopsicosocial. Revista Latinoamericana de Psicología, 33, 117-130.

Wachirawat, W., Hanucharurnkul, S., Suriyawongpaisal, P., Boonyapisit, S., Levenstein, S., Jearanaisilavong, J., Atisook, K., Boontong, T., y Theerabutr, C. (2003). Stress, but not Helicobacter pylori, is associated with peptic ulcer disease in a Thai population. Journal of the Medical Association of Thailand, 86, 672-685.

Weiner, H., Thaler, M., Reiser, M.F., y Mirsky, I.A. (1957). Etiology of duodenal ulcer. I. Relation of specific psychological characteristics to rate of gastric secretion (serum pepsinogen). Psychosomatic Medicine, 19, 1-10. 